\title{
O MODELO DIFUSIVO-CONVECTIVO APLICADO À SECAGEM DE PÊRA UTILIZANDO O MÉTODO DA COLOCAÇÃO ORTOGONAL
}

\author{
T. B. SOUZA, M. R. SIMÕES, C. F. M. MOREJON, M. F. P. MOREIRA* \\ Universidade Estadual do Oeste do Paraná, Departamento de Engenharia Química \\ *e-mail: marcosfpmoreira@gmail.com
}

\begin{abstract}
RESUMO
O objetivo deste trabalho foi avaliar o modelo difusivo-convectivo na secagem da pêra utilizando o método da colocação ortogonal para a solução do modelo. Os números de Biot efetivos de calor e massa $\left(\mathrm{Bi}_{\mathrm{H}}\right.$ e $\left.\mathrm{Bi}_{\mathrm{M}}\right)$ foram determinados pelo ajuste dos valores de umidade e temperatura calculados aos valores obtidos experimentalmente. A secagem foi conduzida em estufa de convecção natural nas temperaturas de $40^{\circ} \mathrm{C}$ e $80^{\circ} \mathrm{C}$ e em um secador de convecção forçada com escoamento paralelo de ar a $40^{\circ} \mathrm{C}$ e $1 \mathrm{~m} / \mathrm{s}$. As amostras de pêra foram cortadas em forma circular com aproximadamente $5 \mathrm{~cm}$ de diâmetro e $5 \mathrm{~mm}$ de espessura e secas por ambos os lados. Verificou-se que o modelo difusivo-convectivo apresentou bom ajuste aos resultados experimentais tanto em condições de Biot efetivo de massa acima como abaixo de 0,1 .
\end{abstract}

\section{INTRODUÇÃO}

As frutas são uma fonte complementar muito importante na dieta dos seres humanos. A pêra (Pyrus spp.), fruto da pereira, é uma das frutas mais consumidas no mundo (Lin e Harnly, 2008). A pêra é produzida por vários países ao redor do mundo como China, Itália, Estados Unidos, Espanha, Argentina e Brasil.

A produção brasileira de pêra de 2001 a 2005 foi de 20.230 toneladas por ano em média, sendo o estado do Rio Grande do Sul o maior produtor do país, com $45,7 \%$ do total produzido, seguido pelos estados de São Paulo, com 22,8\%, e Paraná, com 10,9\% (Fioravanço, 2007).

Sabe-se que as frutas sofrem um processo de deterioração devido ao alto conteúdo de umidade, cerca de $90 \%$, e a secagem é ainda hoje um processo muito empregado para a preservação desses alimentos (Ferreira et al., 2002).

A fim de desenvolver processos adequados de secagem ou otimizar os já existentes, torna-se de fundamental importância o estudo a respeito dos modelos matemáticos que descrevem o fenômeno de secagem.

Vários modelos para secagem em camada fina podem ser encontrados na literatura. Eles podem ser divididos em modelos empíricos e teóricos.

Um dos modelos empíricos mais conhecidos é o famoso modelo de Lewis (Lewis, 1921), o qual é similar à Lei do Resfriamento de Newton.

Dentre os modelos teóricos mais utilizados está o modelo difusivo (Crank, 1956). Uma variação muito utilizada do modelo difusivo com respeito a materiais que encolhem durante o processo de secagem, como é o caso das frutas, é o modelo difusivo com encolhimento (Kechaou e Roques, 1989; Viollaz e Suarez, 1991).

Ainda com respeito a materiais que apresentam o fenômeno de encolhimento durante a secagem, Carneiro (1995) propôs um modelo difusivo-convectivo onde 
considera que parte da saída de umidade do material é facilitada pelo seu encolhimento. Esse modelo foi aplicado à secagem de peixe.

O objetivo deste trabalho foi avaliar modelo difusivo-convectivo na secagem de pêra utilizando $\mathrm{o}$ método da colocação ortogonal para a solução do modelo. Foram testadas condições de convecção natural e convecção forçada.

\section{FORMULAÇÕES UTILIZADAS} NESTE TRABALHO PARA AS TRANSFERÊNCIAS DE MASSA E DE CALOR

\subsection{Transferência de Massa}

$\mathrm{O}$ balanço diferencial de massa utilizado neste trabalho foi baseado no balanço difusivo-convectivo proposto por Carneiro (1995), mas como condição de contorno levou-se em consideração a resistência externa à transferência de massa. $\mathrm{O}$ balanço diferencial de massa é dado por:

$$
\begin{aligned}
& \frac{\partial \mathrm{X}}{\partial \mathrm{t}}=-\frac{\partial \mathrm{n}_{\mathrm{D}}}{\partial \mathrm{z}}-\frac{\partial \mathrm{n}_{\mathrm{c}}}{\partial \mathrm{z}} \\
& \mathrm{n}_{\mathrm{D}}=-\mathrm{D}_{\mathrm{FF}} \frac{\partial \mathrm{X}}{\partial \mathrm{z}} \\
& \mathrm{n}_{\mathrm{c}}=-\mathrm{v} \cdot \mathrm{X}
\end{aligned}
$$

Adimensionalizando a umidade, o tempo e a coordenada axial e considerando que a difusividade efetiva é constante e que a velocidade não varia com a posição tem-se que:

$$
\frac{\partial \mathrm{W}}{\partial \mathrm{Fo}_{\mathrm{M}}}=\frac{\partial^{2} \mathrm{~W}}{\partial \xi^{2}}+\mathrm{Pe}_{\mathrm{s}} \frac{\partial \mathrm{W}}{\partial \xi}
$$

As condições empregadas na formulação foram as seguintes:

(condição inicial)

$$
\mathrm{W}\left(\xi, \mathrm{Fo}_{\mathrm{s}}=0\right)=1
$$

(condição de simetria)

$\left.\frac{\partial \mathrm{W}}{\partial \xi}\right|_{\xi-0}=0$

(condição de contorno)

$$
\begin{aligned}
& \mathrm{n}_{\left.\mathrm{c}\right|_{\xi=1}}+\left.\mathrm{n}_{\mathrm{D}}\right|_{\xi=1}=\mathrm{k}_{\mathrm{m}}\left(\left.\mathrm{X}\right|_{\xi=1}-\overline{\mathrm{X}}_{\mathrm{E}}\right) \text { ou } \\
& \left.\frac{\partial \mathrm{W}}{\partial \xi}\right|_{\xi=1}=-\left.\mathrm{Bi}_{\mathrm{M}} \cdot \mathrm{W}\right|_{\xi=1}-\mathrm{Pe}_{\mathrm{M}} \cdot\left(\left.\mathrm{W}\right|_{\xi=1}+\frac{\overline{\mathrm{X}}_{\mathrm{E}}}{\mathrm{X}_{\mathrm{o}}-\overline{\mathrm{X}}_{\mathrm{E}}}\right)
\end{aligned}
$$

Nessa formulação "v" é a velocidade de encolhimento do material responsável pelo fluxo convectivo de umidade.

Para valores do número de Biot efetivo de massa $\left(\mathrm{Bi}_{\mathrm{M}}\right)$ maiores do que 0,1 a resistência externa à transferência de massa pode ser assumida como desprezível de acordo com Sander et al. (2001). Nessas situações então, pode ser assumido que a umidade na superfície do material é constante e a formulação passa a apresentar solução analítica, sendo a umidade média do material em função do tempo dada por (Carneiro, 1995):

$$
\overline{\mathrm{W}}=\frac{2 \cdot \mathrm{L}_{\circ}}{\mathrm{L}} \sum_{n=1}^{\infty}\left\{\mathrm{A}_{\mathrm{n}} \cdot \exp \left[-\left(\alpha_{\mathrm{n}}^{2}+\frac{\mathrm{Pe}_{\mathrm{M}}^{2}}{4} \cdot \frac{\mathrm{L}_{\mathrm{o}}^{2}}{\mathrm{~L}^{2}}\right) \mathrm{Fo}_{\mathrm{M}} \cdot \frac{\mathrm{L}^{2}}{\mathrm{~L}_{\mathrm{o}}^{2}}\right]\right\}
$$

$$
\mathrm{A}_{\mathrm{n}}=\frac{\operatorname{sen}^{2}\left(2 \alpha_{\mathrm{n}} \mathrm{L} / \mathrm{L}_{\mathrm{o}}\right) \exp \left(\mathrm{Pe}_{\mathrm{N}} / 2\right)}{\left(\mathrm{Pe}_{\mathrm{w}}\right)+2 \cos ^{2}\left(\alpha_{\mathrm{n}} \mathrm{L} / \mathrm{L}_{\mathrm{o}}\right)} \cdot \frac{\exp \left(-\mathrm{Pe}_{\mathrm{N}} / 2\right)-2 \cos \left(\alpha_{\mathrm{n}} \mathrm{L} / \mathrm{L}_{\mathrm{o}}\right)}{\left(\mathrm{Pe}_{\mathrm{N}} \mathrm{L}_{\mathrm{o}} / \mathrm{L}\right)}
$$

$$
\mathrm{Pe}_{\mathrm{M}}=\frac{\mathrm{v} \cdot \mathrm{L}}{\mathrm{D}_{\mathrm{FF}}}
$$

onde os auto-valores $\alpha_{\mathrm{n}}$ são as raízes positivas da equação:

$\alpha_{n} \mathrm{~L} / \mathrm{L}_{\mathrm{o}}=-\frac{P \mathrm{P}_{\mathrm{s}}}{2} \tan \left(\alpha_{\mathrm{n}} \mathrm{L} / \mathrm{L}_{\mathrm{o}}\right)$

e se encontram nos quadrantes pares do círculo trigonométrico. 


\subsection{Transferência de Calor}

A formulação da transferência de calor utilizada neste trabalho teve por base um balanço diferencial de energia difusivoconvectivo dado por:

$\rho_{\mathrm{ss}} \frac{\partial\left[\mathrm{Cp} \cdot\left(\mathrm{T}-\mathrm{T}_{\mathrm{RF}}\right)\right]}{\partial \mathrm{t}}=-\frac{\partial \mathrm{q}_{\mathrm{coND}}}{\partial \mathrm{z}}-\rho_{\mathrm{ss}} \frac{\partial \mathrm{q}_{\mathrm{u}}}{\partial \mathrm{z}}$

onde

$$
\begin{aligned}
& \mathrm{Cp}=\mathrm{Cp}_{\mathrm{ss}}+\mathrm{X} \cdot \mathrm{Cp} \mathrm{p}_{\mathrm{gum}} \\
& \mathrm{q}_{\mathrm{CoND}}=-\mathrm{k}_{\mathrm{FF}} \frac{\partial \mathrm{T}}{\partial \mathrm{z}} \\
& \mathrm{q}_{\mathrm{s}}=\left(\mathrm{n}_{\mathrm{c}}+\mathrm{n}_{\mathrm{D}}\right) \cdot C \mathrm{p}_{\mathrm{gagu}} \cdot\left(\mathrm{T}-\mathrm{T}_{\mathrm{RFF}}\right)
\end{aligned}
$$

Considerando constantes a condutividade térmica efetiva $\left(\mathrm{k}_{\mathrm{EF}}\right)$ e o calor específico da água, tomando a temperatura de referência como a temperatura inicial do material, considerando que a velocidade não é função da posição e adimensionalizando a temperatura e a coordenada axial tem-se que:

$$
\begin{aligned}
& \rho_{\mathrm{ss}} \frac{\partial(\mathrm{Cp} \cdot \theta)}{\partial \mathrm{t}}=\mathrm{k}_{\mathrm{FF}} \frac{\partial^{2} \theta}{\partial \mathrm{z}^{2}}+\ldots \\
& \ldots+\rho_{\mathrm{Ss}} \cdot \mathrm{Cp}_{\mathrm{g \textrm {gga }}}\left[\frac{\mathrm{v}}{\mathrm{L}} \frac{\partial(\mathrm{X} \cdot \theta)}{\partial \xi}+\frac{\mathrm{D}_{\mathrm{FF}}}{\mathrm{L}^{2}} \frac{\partial}{\partial \xi}\left(\theta \frac{\partial X}{\partial \xi}\right)\right]
\end{aligned}
$$
foram:

As condições utilizadas na formulação

(condição inicial)

$\theta(\xi, \mathrm{t}=0)=0$

(condição de simetria)

$$
\left.\frac{\partial \theta}{\partial \xi}\right|_{\xi=0}=0
$$

(condição de contorno)

$$
\begin{aligned}
& \left.\frac{\partial \theta}{\partial \xi}\right|_{\xi=-1}=\mathrm{Bi}_{\mathrm{H}}\left(1-\left.\theta\right|_{\xi=1}\right)-\ldots \\
& \ldots\left(\frac{\Delta \mathrm{H}_{\mathrm{VAP}}}{\mathrm{T}_{\infty}-\mathrm{T}_{\mathrm{i}}}+\left.\mathrm{C} \mathrm{p}_{\mathrm{ig \textrm {gua }}} \cdot \theta\right|_{\xi=1}\right) \cdot\left(\left.\mathrm{n}_{\mathrm{c}}\right|_{\xi=1}+\mathrm{n}_{\mathrm{D} \mid \xi=1}\right) \cdot \frac{\mathrm{L} \cdot \rho_{\mathrm{SS}}}{\mathrm{k}_{\mathrm{BF}}}
\end{aligned}
$$

$\mathrm{O}$ calor latente de vaporização $\left(\Delta \mathrm{H}_{\mathrm{VAP}}\right)$ foi tomado como função da temperatura na superfície da amostra e o calor específico $(\mathrm{Cp})$ e a massa específica de sólido seco ( $\left.\rho_{\mathrm{SS}}\right)$ da amostra como funções da umidade. $\mathrm{O}$ calor específico da água foi tomado igual a 4186 $\mathrm{J} /(\mathrm{kg} . \mathrm{K})$. O calor específico do sólido seco $(\mathrm{Cp}$ Ss) foi considerado igual a $1500 \mathrm{~J} /(\mathrm{kg} . \mathrm{K}) \mathrm{e}$ a massa específica de sólido seco da pêra como sendo (Guiné e Castro, 2003):

$\rho_{\mathrm{SS}}=[1079+299,6 \cdot \exp (-2,6 . X)] /(1+X)$

\subsection{Solução Numérica das Formulações de Transferência de Massa e de Calor}

As formulações de transferência de massa e de calor utilizadas neste trabalho foram resolvidas pelo método da colocação ortogonal.

Os pontos de colocação $\left(\mathrm{x}_{\mathrm{j}}\right)$ utilizados foram as raízes do polinômio de Jacobi sendo $\alpha=0$ e $\beta=-0,5$. Foram utilizados cinco pontos internos de colocação, o que se mostrou mais do que suficiente em todas as condições avaliadas.

O sistema de equações diferenciais ordinárias geradas foi resolvido pelo método de Runge-Kutta de $4^{\text {a }}$ ordem.

Para a obtenção dos $\mathrm{w}_{\mathrm{j}}$ 's, que representam os pesos das quadraturas nos pontos de colocação $x_{j}$ 's, a seguinte fórmula de quadratura para simetria plana foi avaliada:

$\int_{0}^{1} \mathrm{f}(\mathrm{x}) \mathrm{dx}=\sum_{\mathrm{j}=1}^{N+1} w_{j} . \mathrm{f}\left(\mathrm{x}_{\mathrm{j}}\right)$

A função $\mathrm{f}(\mathrm{x})$ proposta como aproximação da variável em estudo foi dada por: 
$f(x)=\sum_{i=1}^{N+1} d \cdot x^{2 \cdot 2}$

onde os valores de $\mathrm{d}_{\mathrm{i}}$ variavam com o tempo.

Os valores médios dos adimensionais de umidade e temperatura em um determinado instante de tempo foram calculados por:

$$
\begin{aligned}
& \overline{\mathrm{W}}=\frac{\sum_{j=1}^{N+1}\left[w_{\mathrm{j}} \cdot \mathrm{W}\left(\mathrm{x}_{\mathrm{j}}\right)\right]}{\sum_{j=1}^{N+1} w_{\mathrm{j}}} \\
& \bar{\theta}=\frac{\sum_{j=1}^{N+1}\left[w_{\mathrm{j}} \cdot \theta\left(\mathrm{x}_{\mathrm{j}}\right)\right]}{\sum_{j=1}^{N+1} w_{\mathrm{j}}}
\end{aligned}
$$

Os parâmetros dos modelos foram obtidos pela minimização das funções dadas por:

$$
\begin{aligned}
& \mathrm{f}_{1}=\sum_{\mathrm{i}=1}^{\mathrm{n}_{\mathrm{\alpha pp}}}\left(\overline{\mathrm{W}}_{\mathrm{exp}, \mathrm{i}}-\overline{\mathrm{W}}_{\mathrm{clac}, \mathrm{i}}\right)^{2} \\
& \mathrm{f}_{2}=\sum_{\mathrm{i}=1}^{\mathrm{n}_{\mathrm{app}}}\left(\bar{\theta}_{\text {exp,i }}-\bar{\theta}_{\text {calc,i }}\right)^{2}
\end{aligned}
$$

Pela hipótese do modelo de transferência de massa não ser afetado pela temperatura, o algoritmo para obtenção dos parâmetros baseou-se primeiramente na minimização de $f_{1}$, obtendo-se então os valores dos parâmetros mássicos. Depois, o algoritmo passou para o passo de minimização da função $\mathrm{f}_{2}$ utilizando os valores dos parâmetros mássicos encontrados no passo anterior. Dessa forma, obtiveram-se então os valores dos parâmetros térmicos. As minimizações das funções $f_{1} \quad e f_{2}$ foram realizadas utilizando o algoritmo "active-set" (Fletcher \& Powell, 1963; Goldfarb, 1970).

Para a utilização do modelo representado pela Equação 5 foi necessária a obtenção dos auto-valores $\alpha_{n}$ para cada instante de tempo avaliado. Esses valores foram obtidos por inspeções consecutivas dos quadrantes dois e quatro do círculo trigonométrico e a cada ciclo de inspeção dois valores eram obtidos, um no quadrante dois e o outro no quadrante quatro.

Em um determinado instante de tempo, cuja velocidade era "v" e a metade da espessura total do material " $L$ ", calculava-se o valor de F, dado por:

$$
\mathrm{F}=\left|\frac{\alpha_{\mathrm{n}} \mathrm{L} / \mathrm{L}_{\mathrm{o}}}{\tan \left(\alpha_{\mathrm{n}} \mathrm{L} / \mathrm{L}_{\mathrm{o}}\right)}+\frac{\mathrm{Pe}_{\mathrm{M}}}{2}\right|
$$

O valor de F era calculado para 10.000 valores de $\alpha_{n}$ tomados no quadrante de interesse. Assumia-se como raiz da Equação 28 o valor de $\alpha_{n}$ que fornecia o valor de $\mathrm{F}$ mais próximo de zero. Foram determinados 300 valores de $\alpha_{\mathrm{n}}$ para cada instante de tempo analisado.

Todos os cálculos foram realizados com auxílio dos softwares Excel/VBA ${ }^{\circledR}$ e Matlab ${ }^{\circledR}$.

\section{DETERMINAÇÕES EXPERIMENTAIS}

Para a secagem das amostras de pêra utilizaram-se uma estufa de convecção natural e um secador de convecção forçada conforme o esquema apresentado na Figura 1.

Figura 1 - Esquema do módulo de secagem de convecção forçada.

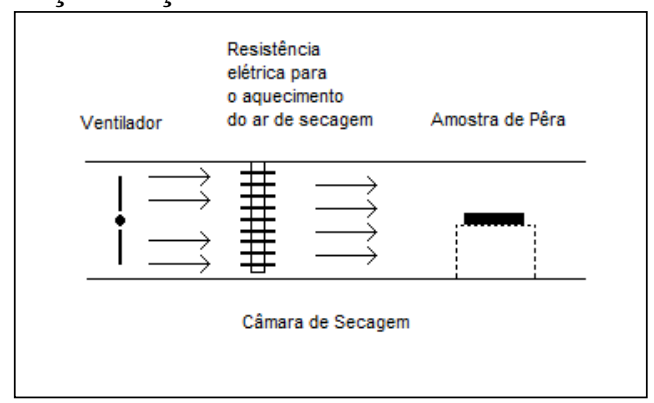

Fonte: Autor (2015).

As amostras de pêra utilizadas no processo de secagem tinham a forma de disco com $5 \mathrm{~cm}$ de diâmetro e $5 \mathrm{~mm}$ de espessura aproximadamente. A amostra de pêra foi colocada sobre uma bandeja perfurada de forma a entrar em contato total com o ar de 
secagem.

$\mathrm{Na}$ secagem em convecção forçada o escoamento era paralelo à amostra, sendo o ar mantido a uma temperatura de $40^{\circ} \mathrm{C}$ e velocidade de $1 \mathrm{~m} / \mathrm{s}$. Na secagem em estufa foram empregadas temperaturas de $40^{\circ} \mathrm{C}$ e $80^{\circ} \mathrm{C}$ medidas próximas à amostra.

A massa e a espessura das amostras eram medidas de trinta em trinta minutos.

Após o final da secagem a amostra era levada para a determinação da "massa seca" segundo o método apresentado por Lees (1980) a fim de se possibilitar o cálculo da umidade do material. $\mathrm{O}$ método basicamente consiste em deixar a amostra por 4 horas em uma estufa a $105^{\circ} \mathrm{C}$, pesando-se a amostra ao final deste tempo, tornando a levá-la para a estufa por mais 30 minutos e pesando-a novamente. Se a massa estiver variando continua-se a secagem de 30 em 30 minutos até que a massa permaneça constante.

A determinação da temperatura da amostra em função do tempo foi feita em experimentos à parte em estufa a $40^{\circ} \mathrm{C}$. Foi colocado um termopar no centro da amostra. O diâmetro da ponta do termopar era de 3 $\mathrm{mm}$. Foram registradas as temperaturas de minuto a minuto durante 7 horas.

Os experimentos foram conduzidos com réplicas para se verificar a reprodutibilidade dos dados experimentais obtidos.

\section{RESULTADOS E DISCUSSÕES}

\subsection{Espessura do Material e Velocidade de Encolhimento}

Verificou-se que todas as amostras apresentaram um encolhimento típico ao apresentado na Figura 2.

A espessura da amostra variou com relação ao tempo na forma de um polinômio de segundo grau para todas as condições estudadas. A variância explicada $\left(\mathrm{r}^{2}\right)$ para os polinômios obtidos ficou entre $98,2 \%$ a $99,4 \%$.

A velocidade de encolhimento das amostras foi tomada a partir da derivação do polinômio com relação ao tempo. A Figura 3 apresenta os valores de velocidade obtidos nas condições estudadas.

Figura 2 - Comprimento adimensional em função do tempo para a secagem a $80^{\circ} \mathrm{C}$ em estufa.

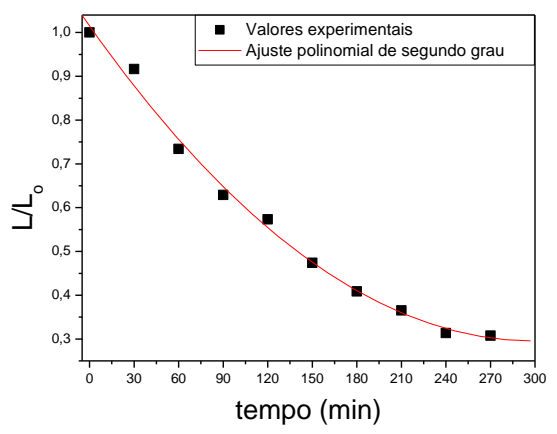

Fonte: Autor (2015).

Figura 3 - Velocidade de encolhimento da pêra em função do tempo.



Fonte: Autor (2015).

A partir da Figura 3 foi possível verificar que as velocidades de encolhimento do material nas condições de secagem em estufa a $80^{\circ} \mathrm{C}$ e na secagem em convecção forçada $\left(40^{\circ} \mathrm{C}\right.$ e $\left.1 \mathrm{~m} / \mathrm{s}\right)$ foram muito próximas entre si, enquanto que a velocidade de encolhimento na secagem em estufa a $40^{\circ} \mathrm{C}$ apresentou valores inferiores às velocidades das outras condições avaliadas.

\subsection{Parâmetros Mássicos}

Considerando a espessura e a velocidade variáveis com a umidade média do material, e, por conseguinte, variáveis com o 
tempo, ajustaram-se as umidades calculadas às obtidas experimentalmente e foram obtidos os parâmetros mássicos $\mathrm{D}_{\mathrm{EF}}, \mathrm{k}_{\mathrm{m}}, \mathrm{Bi}_{\mathrm{M}}$, e $\mathrm{Pe}_{\mathrm{M}}$. Todos os valores dos parâmetros mássicos estão apresentados na Figura 4, sendo " $\mathrm{L}_{0}$ " o comprimento característico utilizado nos números de Biot e Péclet de massa. Também é apresentada a velocidade média de encolhimento do material tomada como:

$\overline{\mathrm{v}}=\frac{\int \mathrm{v}(\mathrm{t}) \cdot \mathrm{dt}}{\Delta \mathrm{t}}=\frac{\mathrm{v}(\mathrm{t}=0)}{2}$

Inicialmente pôde se verificar que na secagem em estufa a $40^{\circ} \mathrm{C}$ o número de Biot efetivo de massa $\left(\mathrm{Bi}_{\mathrm{M}}\right)$ foi inferior a 0,1 , indicando a importância da resistência externa $\left(1 / \mathrm{k}_{\mathrm{m}}\right)$ no fenômeno da transferência de massa frente à resistência interna $\left(\mathrm{L}_{\mathrm{o}} / \mathrm{D}_{\mathrm{EF}}\right)$. Com o aumento da temperatura para $80^{\circ} \mathrm{C}$ na secagem em estufa, o valor de Biot foi superior a 0,1 . O aumento da temperatura favoreceu a diminuição da resistência interna $\left(\mathrm{L}_{\mathrm{o}} / \mathrm{D}_{\mathrm{EF}}\right)$ como era esperado, mas favoreceu mais ainda a diminuição da resistência externa $\left(1 / \mathrm{k}_{\mathrm{m}}\right)$, o que permite concluir que a umidade na superfície do material durante todo o processo de secagem pode ser tomada como a própria umidade de equilíbrio do material nessa condição.

Figura 4 - Parâmetros mássicos e velocidade de encolhimento em função das condições de secagem.

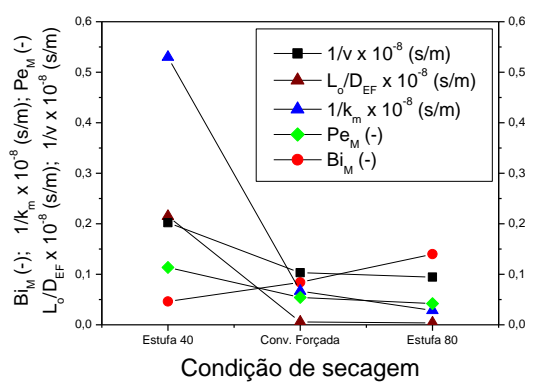

Fonte: Autor (2015).

Em relação à influência da velocidade do ar de secagem sobre o número de Biot, verificou-se que houve um aumento do número de Biot ao se passar da secagem em estufa a $40^{\circ} \mathrm{C}$ para a secagem em convecção forçada $\left(40^{\circ} \mathrm{C}\right.$ e $\left.1 \mathrm{~m} / \mathrm{s}\right)$. O número de Biot passou de 0,05 para 0,09 , ou seja, a utilização de convecção forçada com velocidade do ar de $1 \mathrm{~m} / \mathrm{s}$ tornou a resistência externa $\left(1 / \mathrm{k}_{\mathrm{m}}\right)$ à transferência de massa muito pequena frente à resistência interna $\left(\mathrm{L}_{\mathrm{o}} / \mathrm{D}_{\mathrm{EF}}\right)$, apesar da resistência interna ter diminuído também.

Quanto ao número de Péclet efetivo de massa $\left(\mathrm{Pe}_{\mathrm{M}}\right)$, que representa a razão entre as resistências internas difusiva $\left(\mathrm{L}_{\mathrm{o}} / \mathrm{D}_{\mathrm{EF}}\right)$ e convectiva $(1 / \mathrm{v})$ à transferência de massa, verificou-se que houve uma diminuição de cerca de $63 \%$ em seu valor com o aumento da temperatura na secagem em estufa, indicando que o aumento da temperatura causou mais a diminuição da resistência difusiva $\left(\mathrm{L}_{\mathrm{o}} / \mathrm{D}_{\mathrm{EF}}\right)$ do que da resistência convectiva $(1 / \mathrm{v})$, ou seja, ocorreu mais a saída de umidade pelo processo de difusão efetiva do que pelo processo de convecção. Esse mesmo comportamento qualitativo foi encontrado ao se comparar a convecção natural com a convecção forçada à temperatura de $40^{\circ} \mathrm{C}$. Ao se passar da convecção natural para a convecção forçada ocorreu uma diminuição de $53 \%$ no valor do número de Péclet efetivo de massa $\left(\mathrm{Pe}_{\mathrm{M}}\right)$.

\subsection{Qualidade do Ajuste dos Modelos com e sem Resistência Externa à Transferência de Massa}

A Figura 5 apresenta o ajuste fornecido pelos modelos com e sem resistência externa à transferência de massa.

De acordo com a Figura 5, visualmente os ajustes fornecidos pelos modelos com e sem resistência foram semelhantes nas condições de Biot próximo ou superior a 0,1 , casos das secagens em estufa a $80^{\circ} \mathrm{C}$ e em convecção forçada.

Para a secagem em estufa a $40^{\circ} \mathrm{C}$, onde o número de Biot foi de 0,05, a qualidade do 
ajuste do modelo com resistência é visivelmente superior à do modelo sem resistência. $\mathrm{O}$ modelo sem resistência conseguiu uma boa previsão apenas nos instantes iniciais da secagem quando há uma grande quantidade de umidade livre na superfície do material. Cabe salientar que no modelo sem resistência a espessura e a velocidade de encolhimento da amostra foram assumidas como variáveis em relação à umidade média do material, e, por conseguinte, variáveis com relação ao tempo conforme utilizado por Carneiro (1995).

Figura 5 - Adimensional de umidade em função do tempo.

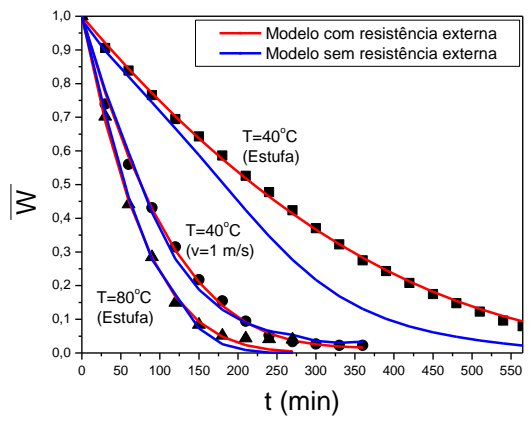

Fonte: Autor (2015).

Nas condições onde os modelos apresentaram desempenhos semelhantes, a difusividade efetiva do modelo com resistência foi cerca de cem vezes a difusividade do modelo sem resistência.

\subsection{Avaliação da Transferência de Calor}

Os dados experimentais de temperatura na secagem da pêra em estufa a $40^{\circ} \mathrm{C}$ estão apresentados na Figura 6, assim como os valores calculados pelo modelo proposto.

De forma geral verificou-se que o modelo conseguiu manter a tendência dos valores experimentais de temperatura em função do tempo obtendo-se um valor de $\mathrm{f}_{2} \mathrm{de}$ 2,4052.

Em relação ao valor da condutividade efetiva da pêra obteve-se um valor de 0,50
W/(m.K), enquanto Guiné et al. (2007) verificaram uma condutividade de 0,177 $\mathrm{W} /(\mathrm{m} . \mathrm{K})$. Essa diferença pode ser explicada por dois motivos, o primeiro seria a baixa sensibilidade da temperatura do material à condutividade térmica efetiva, o que dificulta a estimativa do parâmetro com precisão; a linha azul na Figura 6 representa as temperaturas calculadas com a condutividade térmica efetiva de $0,177 \mathrm{~W} /(\mathrm{m} . \mathrm{K})$. O segundo motivo seria a utilização de modelos matemáticos diferentes para a obtenção da condutividade, pois Guiné et al. (2007) não utilizaram o mesmo modelo deste trabalho.

Figura 6 - Temperatura média adimensional em função do tempo.

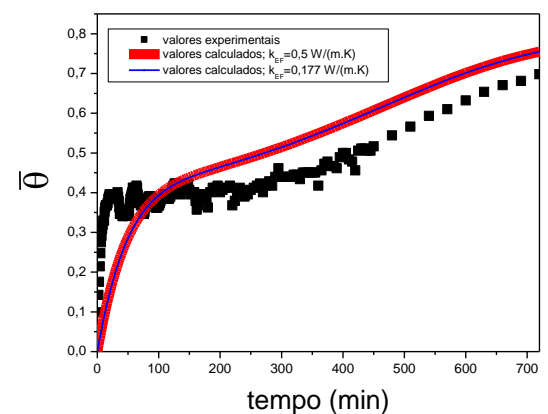

Fonte: Autor (2015).

Quanto aos parâmetros térmicos, a Tabela 1 apresenta os valores obtidos.

Tabela 1 - Valores dos parâmetros térmicos para a secagem em estufa a $40^{\circ} \mathrm{C}$.

\begin{tabular}{cccc}
$\begin{array}{c}\text { Estufa } \\
40^{\circ} \mathrm{C}\end{array}$ & $\mathrm{Bi}_{\mathrm{H}}$ & $\begin{array}{c}\mathrm{k}_{\mathrm{EF}} \\
\left(\mathrm{W} \cdot \mathrm{m}^{-1} \cdot \mathrm{K}^{-1}\right)\end{array}$ & $\begin{array}{c}\mathrm{h} \\
\left(\mathrm{W} \cdot \mathrm{m}^{-2} \cdot \mathrm{K}^{-1}\right)\end{array}$ \\
\cline { 2 - 4 } & 0,023 & 0,50 & 4,2
\end{tabular}

Fonte: Autor (2015).

Quanto ao valor do coeficiente de troca térmica obtido, 4,2 $\mathrm{W} /\left(\mathrm{m}^{2} . \mathrm{K}\right)$, verifica-se na literatura (Holman, 1983) que é um valor coerente para as condições utilizadas experimentalmente.

Quanto ao valor do Biot efetivo de calor, verificou-se um valor abaixo de 0,1 
como está apresentado na Tabela 1, o que indica que o fenômeno pode ser tratado com uma análise concentrada, ou seja, com a temperatura do material não sendo função da posição, pois a resistência condutiva $\left(\mathrm{L}_{\mathrm{o}} / \mathrm{k}_{\mathrm{EF}}\right)$ é desprezível frente à resistência convectiva (1/h). A Figura 7 apresenta o perfil calculado de temperatura da pêra em diferentes instantes de tempo. É possível verificar que a temperatura quase não varia com a posição nem mesmo nos instantes iniciais da secagem, o que é resultado do baixo valor do Biot efetivo de calor.

Figura 7 - Temperatura adimensional em função da posição.

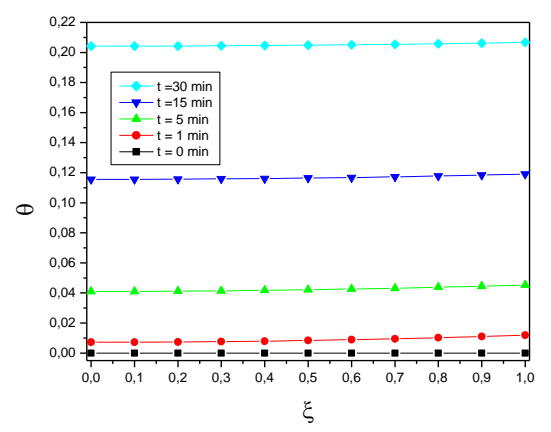

Fonte: Autor (2015).

Com relação aos perfis de umidade, verificou-se, como está apresentado na Figura 8 , que os perfis são acentuados e variam com o tempo. Os perfis são do tipo parabólico na maior parte do tempo, exceto no início e no final da secagem quando se apresentam como perfis planos.

\subsection{Sensibilidade Paramétrica}

A fim de se verificar a importância dos parâmetros mássicos e térmicos $\left(\mathrm{D}_{\mathrm{EF}}, \mathrm{k}_{\mathrm{m}}, \mathrm{k}_{\mathrm{EF}}\right.$, h) estimados neste trabalho foi realizado um estudo sobre a sensibilidade da umidade e da temperatura da pêra a tais parâmetros. Para tanto, tomou-se como tempo de referência o instante $\mathrm{t}=300 \mathrm{~min}$ na secagem em estufa a $40^{\circ} \mathrm{C}$. Variou-se o parâmetro de interesse na faixa de 0,2 a 1,9 vezes o valor estimado "jestimado" (veja os eixos x nas Figuras 9 e 10) mantendo-se os outros parâmetros em seus valores originais e determinaram-se as variações ocasionadas na umidade e temperatura médias da pêra. As sensibilidades da umidade e da temperatura ao parâmetro de interesse " $\mathrm{j}$ " foram calculadas como:

$S_{x}=j \frac{\partial X}{\partial j} \cong j \frac{\Delta X}{\Delta j}$

$S_{\mathrm{r}}=\mathrm{j} \frac{\partial \mathrm{T}}{\partial \mathrm{j}} \cong \mathrm{j} \frac{\Delta \mathrm{T}}{\Delta \mathrm{j}}$

As Figuras 9 e 10 apresentam os resultados obtidos.

Figura 8 - Adimensional de umidade em função da posição.

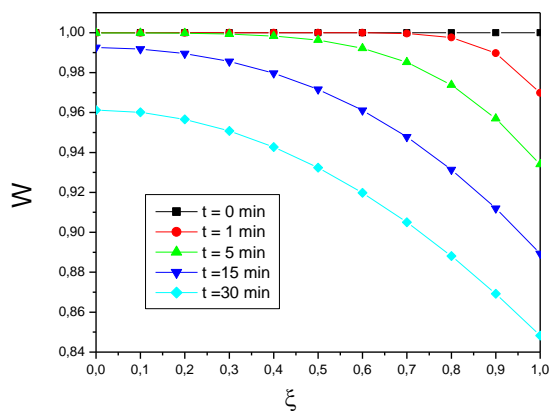

Fonte: Autor (2015).

Figura 9 - Sensibilidade da umidade da pêra aos parâmetros mássicos.

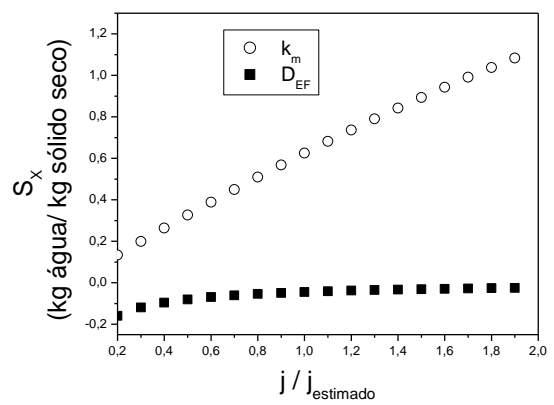

Fonte: Autor (2015).

Como, por hipótese, a formulação da 
transferência de massa foi resolvida sem a necessidade do conhecimento do perfil de temperatura, a umidade média do material não foi sensível a $k_{E F}$ e h. Com relação a $D_{E F}$ e $k_{m}$ verificou-se, conforme apresenta a Figura 9, que a sensibilidade da umidade foi muito maior a $\mathrm{k}_{\mathrm{m}}$ do que à difusividade, ou seja, pequenas mudanças na estimativa de $\mathrm{k}_{\mathrm{m}}$ podem ocasionar grandes modificações na previsão da umidade do material, enquanto que grandes modificações na difusividade causam pequenas modificações na umidade, $o$ que torna problemática a estimativa da $\mathrm{D}_{\mathrm{EF}}$.

Em relação à sensibilidade da temperatura aos parâmetros avaliados, verificou-se, conforme apresenta a Figura 10, que é em relação a $\mathrm{k}_{\mathrm{m}} \mathrm{e}$ $\mathrm{h}$ que a temperatura do material é mais sensível. A sensibilidade da temperatura a $\mathrm{D}_{\mathrm{EF}}$ e $k_{E F}$ foi praticamente nula, ou seja, a temperatura quase não é afetada por esses parâmetros. Adotando valores para $\mathrm{k}_{\mathrm{EF}}$ na faixa de 0,1 a $1,0 \quad \mathrm{~W} /(\mathrm{m} . \mathrm{K})$ obtem-se exatamente os mesmos valores de temperatura como já havia sido discutido em relação à Figura 6.

Figura 10 - Sensibilidade da temperatura da pêra aos parâmetros mássicos e térmicos.

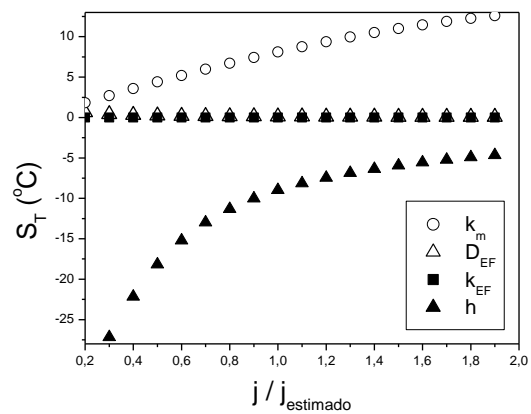

Fonte: Autor (2015).

\section{CONCLUSÕES}

O objetivo do presente trabalho foi avaliar o modelo difusivo-convectivo na secagem da pêra utilizando o método da colocação ortogonal. De maneira geral, o modelo difusivo-convectivo conseguiu obter bons ajustes às medidas experimentais de umidade e de temperatura. Verificou-se que a resistência externa à transferência de massa deve ser considerada na formulação para baixos valores de Biot de massa $(0,05)$. Verificou-se ainda uma baixa sensibilidade da umidade e da temperatura calculadas pelo modelo em relação à $\mathrm{D}_{\mathrm{EF}} \mathrm{e}$ à $\mathrm{k}_{\mathrm{EF}}$.

\section{NOMENCLATURA}

$\mathrm{Bi}_{\mathrm{H}} \quad$ número de Biot efetivo de calor, h.L $/ \mathrm{k}_{\mathrm{EF}}$

$\mathrm{Bi}_{\mathrm{M}}$ número de Biot efetivo de massa,

$$
\mathrm{k}_{\mathrm{m}} \cdot \mathrm{L} / \mathrm{D}_{\mathrm{EF}}
$$

$\mathrm{Cp} \quad$ calor específico da amostra, $\mathrm{J} /(\mathrm{kg} . \mathrm{K})$

$\mathrm{D}_{\mathrm{EF}} \quad$ difusividade efetiva, $\mathrm{m}^{2} / \mathrm{s}$

Cpágua calor específico da água, $\mathrm{J} /(\mathrm{kg} . \mathrm{K})$

$\mathrm{Cp}_{\mathrm{sS}} \quad$ calor específico do sólido seco, J/(kg.K)

$\mathrm{Fo}_{\mathrm{M}}$ número de Fourier efetivo de massa, $\mathrm{D}_{\mathrm{EF} . \mathrm{t} / \mathrm{L}^{2}}$

h coeficiente de troca térmica, $\mathrm{W} /\left(\mathrm{m}^{2} . \mathrm{K}\right)$

$\mathrm{k}_{\mathrm{EF}} \quad$ condutividade térmica efetiva, $\mathrm{W} /(\mathrm{m} . \mathrm{K})$

$\mathrm{k}_{\mathrm{m}} \quad$ coeficiente de transferência de massa, $\mathrm{m} / \mathrm{s}$

L metade da espessura da amostra em um instante de tempo qualquer, $\mathrm{m}$

$\mathrm{L}_{\mathrm{F}} \quad$ metade da espessura da amostra ao final da secagem, $m$

$\mathrm{L}_{\mathrm{o}} \quad$ metade da espessura inicial da amostra, $\mathrm{m}$

$\mathrm{N}$ número de pontos internos de colocação

$\mathrm{Pe}_{\mathrm{M}} \quad$ número de Péclet de massa, v.L/D $\mathrm{D}_{\mathrm{EF}}$

$\mathrm{q}_{\text {cond }}$ calor de condução por unidade de área, $\mathrm{W} / \mathrm{m}^{2}$

$\mathrm{q}_{\mathrm{M}}$ energia transferida devido à migração interna de umidade no material, W.m/kg

$\mathrm{S}_{\mathrm{T}} \quad$ sensibilidade da temperatura, ${ }^{\circ} \mathrm{C}$

$\mathrm{S}_{\mathrm{X}} \quad$ sensibilidade da umidade, b.s.

$\mathrm{t}$ tempo, $\mathrm{s}$

$\mathrm{T}$ temperatura, $\mathrm{K}$

$\mathrm{T}_{\mathrm{i}} \quad$ temperatura inicial, $\mathrm{K}$

$\mathrm{T}_{\infty} \quad$ temperatura do ar de secagem, $\mathrm{K}$

v velocidade de encolhimento, $\mathrm{m} / \mathrm{s}$

$\mathrm{x}_{\mathrm{j}} \quad$ raiz do polinômio de Jacobi 
X umidade local do material, b.s.

$\overline{\mathrm{X}} \quad$ umidade média, b.s.

$\overline{\mathrm{X}}_{\mathrm{F}} \quad$ umidade de equilíbrio, b.s.

$\mathrm{X}_{\mathrm{o}} \quad$ umidade inicial, b.s.

$\mathrm{W}_{\mathrm{j}} \quad$ peso da quadratura em para $\mathrm{x}_{\mathrm{j}}$

W adimensional de umidade, $\left(\mathrm{X}-\overline{\mathrm{X}}_{\mathrm{E}}\right) /\left(\mathrm{X}_{\mathrm{O}^{-}}\right.$ $\overline{\mathrm{X}}_{\mathrm{E}}$ )

$\overline{\mathrm{W}} \quad$ adimensional de umidade médio,

$$
\left(\bar{X}-\bar{X}_{\mathrm{E}}\right) /\left(\mathrm{X}_{\mathrm{O}}-\overline{\mathrm{X}}_{\mathrm{E}}\right)
$$

$\overline{\mathrm{W}}_{\text {смс }}$ adimensional de umidade médio calculado

$\overline{\mathrm{W}}_{\mathrm{ExP}} \quad$ adimensional de umidade médio experimental

$\mathrm{z} \quad$ coordenada axial, $\mathrm{m}$

\section{Letras Gregas}

$\Delta \mathrm{H}_{\mathrm{va}}$ calor latente de vaporização da água, J/kg

$\theta$ temperatura local adimensional $\left[=\left(\mathrm{T}-\mathrm{T}_{\mathrm{i}}\right) /\left(\mathrm{T}_{\infty}-\mathrm{T}_{\mathrm{i}}\right)\right]$

$\bar{\theta} \quad$ temperatura média adimensional $\left[=\left(\overline{\mathrm{T}}-\mathrm{T}_{\mathrm{i}}\right) /\left(\mathrm{T}_{\infty}-\mathrm{T}_{\mathrm{i}}\right)\right]$

$\bar{\theta}_{\text {cuc }} \quad$ temperatura média adimensional calculada

$\bar{\theta}_{\text {Ехр }} \quad$ temperatura média adimensional experimental

$\rho_{\mathrm{SS}} \quad$ massa específica do sólido seco, $\mathrm{kg}$ de sólido $\mathrm{seco} / \mathrm{m}^{3}$ de volume da amostra

$\xi \quad$ adimensional de espessura (=z/L)

\section{REFERÊNCIAS}

CARNEIRO, M. J. M. Modelagem Matemática da Secagem de Pescado. 1995. 74P. Dissertação (Mestrado em Engenharia de Alimentos) Universidade Estadual de Campinas, Campinas, 1995.

CRANK, J. The Mathematics of Diffusion. Londres: Oxford University Press Amen dHouse, 1956.

FERREIRA, D.; GUYOT, S.; MARNET, N. DELGADILLO, I.; RENARD, C. M. G. C.; COIMBRA, M. A. Composition of Phenolic
Compounds in a Portuguese Pear (Pyrus communis L. Var. S. Bartolomeu) and Changes after SunDrying. Journal of Agricultural and Food Chemistry, v.50, p.4537-4544, 2002.

FIORAVANÇO, J. C. The Pear Trees Plantation in Brazil: Economic Situation and Barriers to Their Growth. Economic Information, v.37, p.52-60, 2007.

FLETCHER, R.; POWELL, M. J. D. A Rapidly Convergent Descent Method for Minimization. Computer Journal, v.6, p.163-168, 1963.

GOLDFARB, D. A Family of Variable Metric Updates Derived by Variational Means. Mathematics of Computing, v.24, p.23-26, 1970.

GUINÉ, R.P.F.; CASTRO, J.A.A.M. Analysis of moisture content and density of pears during drying, Drying Technology, v.21(3), p.581-591, 2003.

GUINÉ, R.P.F.; RODRIGUES, A.E.; FIGUEIREDO, M.M. Modelling and Simulation of Pear Drying. Applied Mathematics and Computation, v.192, p.69-77, 2007.

HOLMAN, J. P. Transferência de Calor. São Paulo: McGraw-Hill, 1983.

KECHAOU, N.; ROQUES, M. A Variable Diffusivity Model for Drying of Highly Deformable Material. In: Mujumdar, A. Drying'89. 1989, New York, Hemisphere Publishing Company, 1989, p.332-338.

LEES, R. Analisis de los alimentos: Métodos analíticos y de control de calidad. Zaragoza: Editorial Acribia, 1980.

LEWIS, W. K. The Rate of Drying of Solids Materials. The Journal of I.\&E.Ch., v.13, p.427-432, 1921.

LIN, L.; HARNLY, J. M. Phenolic Compounds and Chromatographic Profiles of Pear Skins (Pyrus spp.). Journal of Agricultural and Food Chemistry, v.56, p.9094-9101, 2008.

SANDER, A.; KARDUM, J. P.; SKANSI, D. Transport Properties in Drying of Solids. Chemical and Biochemical Engineering Quarterly, v.15(3), p.131-137, 2001.

VIOLLAZ, P.; SUAREZ, C. Shrinkage effect on drying behavior of potato slabs. Journal of Food Engineering, v.13, p.103-114, 1991. 